\title{
Site Characterization and Detector Development for the Greenland Neutrino Observatory
}

\author{
Stephanie A. Wissel ${ }^{*}{ }^{a}$ J. Avva, ${ }^{b}$ K. Bechtol, ${ }^{b}$ C. Deaconu, ${ }^{b}$, P. Gorham, ${ }^{c}$ C. Miki, ${ }^{c}$ \\ R. Nichol, ${ }^{d}$ A. Romero-Wolf, ${ }^{e}$ C. Schlupf,${ }^{a}$ D. Saltzberg, ${ }^{a}$ A. Vieregg, ${ }^{b}$, and \\ G. Varner, ${ }^{c}$
}

${ }^{a}$ Dept. of Physics and Astron., Univ. of California, Los Angeles, Los Angeles, CA 90095, USA.

${ }^{b}$ KICP, Univ. of Chicago, Chicago, IL 60637, USA.

${ }^{c}$ Dept. of Physics and Astron., Univ. of Hawaii, Manoa, HI 96822, USA

${ }^{d}$ Dept. of Physics and Astron., University College London, London, United Kingdom.

e Jet Propulsion Laboratory, Pasadena, CA 91109, USA.

Email: swisselephysics.ucla.edu

The PeV neutrinos discovered by IceCube are of astrophysical origin, and their progenitors could be any of several source classes, including active galactic nuclei, gamma-ray bursts, or pulsars. Such high-energy accelerators would produce neutrinos up to hundreds of $\mathrm{PeV}$, which motivates the development of neutrino telescopes with the sensitivity, energy resolution, and pointing resolution required to distinguish among models of the IceCube neutrinos as well as cosmogenic neutrinos. Radio detection of Askaryan radiation from neutrino showers in ice is well-suited to the detection of the highest energy neutrinos, with degree-scale pointing resolution and the ability to build sparse arrays, but the energy threshold of current experiments is currently set by the temperature of the ice. The uncorrelated thermal noise can be reduced by combining the signals from several antennas in a phased array. We report here on a June 2015 trip to Summit Station In Greenland for testing a phased array of dipoles, as well as the sensitivity of the array and background measurements of the site and discuss prospects for the Greenland Neutrino Observatory (GNO).

The 34th International Cosmic Ray Conference,

30 July- 6 August, 2015

The Hague, The Netherlands

\footnotetext{
* Speaker.
} 


\section{Introduction}

High-energy neutrinos carry information about distant astrophysical sources otherwise unavailable, making them a unique messenger particle. A background flux of the highest energy neutrinos $\left(10^{17} \mathrm{eV}\right)$, so-called GZK neutrinos, is expected as a result of the interaction of the highestenergy cosmic rays with the cosmic microwave background [1], but individual sources may also produce neutrinos at that energy.

A promising technique for the detection of the highest energy neutrinos is through measuring the impulsive radio emission from neutrino-induced particle showers in ice. The radio frequency (RF) emission arises from a slight charge imbalance built up as a particle shower develops in a dense dielectric, the Askaryan effect [2,3]. Such emission has been measured and characterized at beam-line experiments $[4,5,6]$. This technique has been exploited by several experiments in Antarctica, including ANITA [7], ARA [8], and ARIANNA [9]. These experiments have thresholds $>50 \mathrm{PeV}$, however, recent studies have shown improved sensitivity to the PeV energy scale using phased arrays of antennas [10,11], which would complement studies of the few-PeV astrophysical neutrino signal discovered by IceCube [12].

We describe the 2015 deployment season in which an array of up to eight antennas was deployed at Summit Station in Greenland. The primary goals of the trip were to explore the radiofrequency environment of Summit Station and to test several configurations of a phased array of dipoles.

\section{GNO Site}

Located approximately $100 \mathrm{~m}$ from Summit Station $\left(72^{\circ} 35^{\prime} 46^{\prime \prime} N, 38^{\circ} 25^{\prime} 19^{\prime \prime} W\right)$, the site for the GNO prototype has several advantages related to both the science and the logistics. As our goals for the 2015 deployment season included site surveying, we first selected Greenland to assess its viability as a UHE neutrino telescope site. We next selected the test site for the DISC (Deep IceSheet Coring) borehole drill to eliminate the need for drilling. The DISC borehole is an available full-depth borehole on the edge of Summit Station.

The National Science Foundation sponsors Summit Station year-round as a research station and provides on-site science support. The layout of Summit Station is shown in Fig. 2. There is a snow runway that allows for cargo and personnel delivery via LC-130 Hercules flights. Solar power is available 10 months a year. The station lies at the apex of the Greenlandic ice sheet, over more than $3 \mathrm{~km}$ of ice. The ice depth is comparable to the South Pole (10\% deeper), although the firn depth of $100 \mathrm{~m}$ is shallower $50 \%$ shallower .

In June 2013, we measured the radiation attenuation length of the ice by comparing the reflected power reflected off the bed rock to the power transmitted by an LPDA [13]. The attenuation length averaged over all depths is $947_{-85}^{+92} \mathrm{~m}$ at $75 \mathrm{MHz}$. The systematic uncertainty on the ground bounce is dominated by the unknown reflection coefficient of the ice-bedrock interface. Combining the depth-averaged attenuation length with measured temperature profiles of the Summit Station and the measured temperature dependence of the attenuation length on temperature, we can construct a depth profile of the attenuation length, shown in Fig. 2. This allows us to calculate the average field attenuation length over the upper $1.5 \mathrm{~km}$ of ice, where we expect an antenna array 


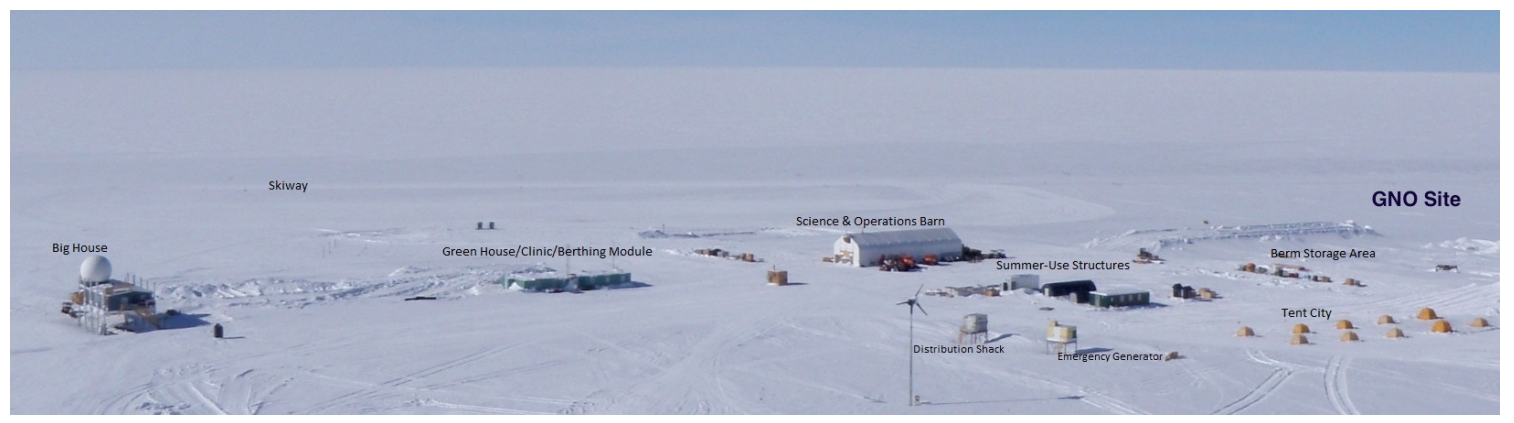

(a)

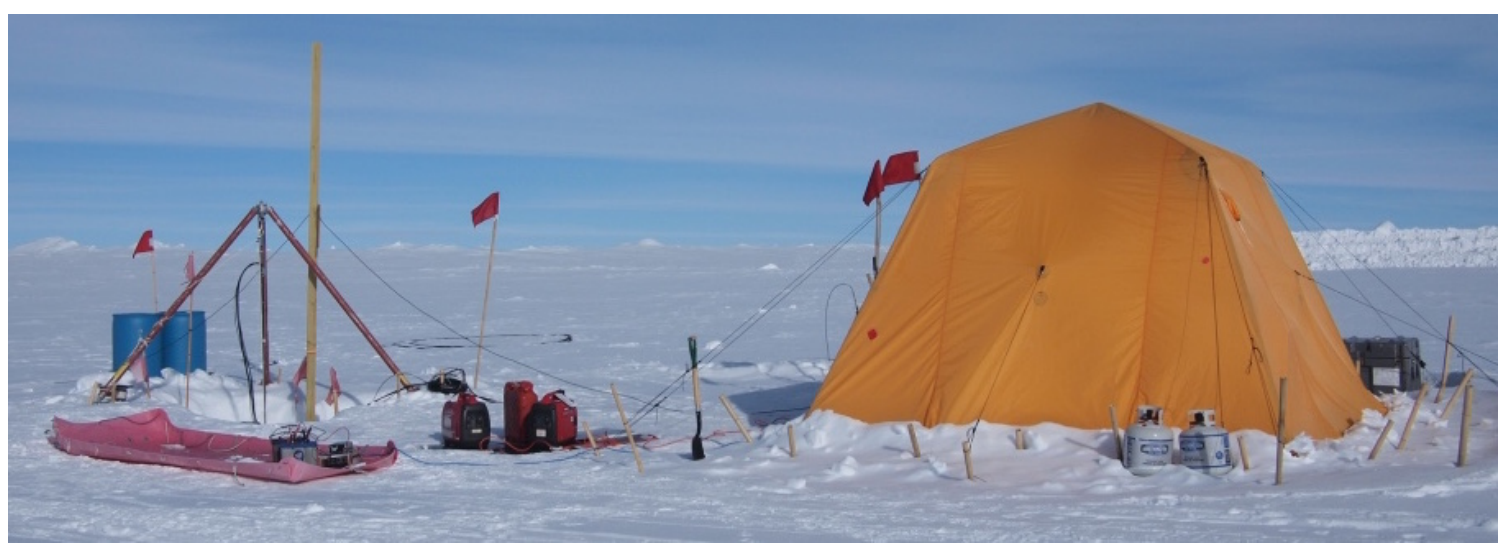

(b)

Figure 1: (a) Overhead view of Summit Station in Greenland (b) The GNO prototype site at the DISC borehole.

deployed below the firn to be most sensitive to neutrino interactions. At $75 \mathrm{MHz}$, the average field attenuation length is $1149_{-103}^{+112} \mathrm{~m}$. Extrapolated to $300 \mathrm{MHz}$ for direct comparison with previous measurements [14, 15, 16], the attenuation length in the upper $1.5 \mathrm{~km}$ is $1022_{-253}^{+230} \mathrm{~m} \mathrm{[13].}$

In addition to the full depth borehole, the GNO site (Fig. 2) includes a $2 \mathrm{~m}$ tripod that acts as a gantry for a $1000 \mathrm{lb}$. capacity winch. Aside from their primary purpose of lowering the antenna array, the gantry and winch were used in conjunction with a deep-well pump to evacuate Isopar-K fluid in the borehole. The fluid was originally added after drilling to prevent the hole from collapsing in on itself and to improve thermal coupling between the hole and the ice. After evacuation, the final surface level of the Isopar-K was $96 \mathrm{~m}$. We deployed our antennas just above this depth, because this is the depth, informed by simulations [10, 11], corresponding to an optimal geometric accep-

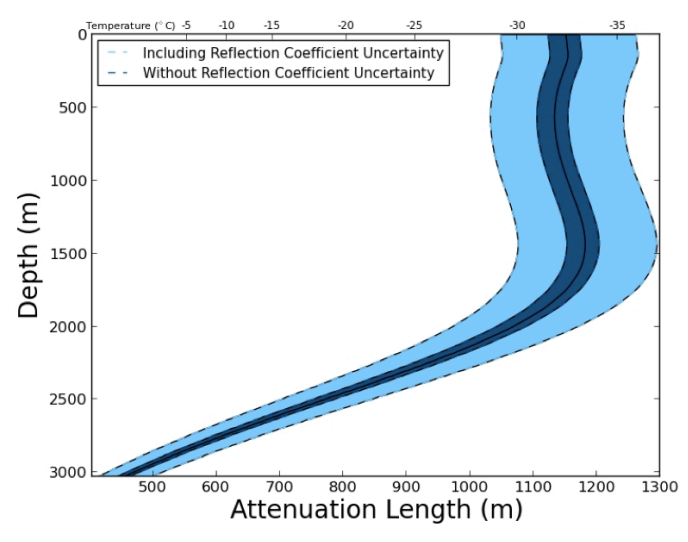

Figure 2: Ice attenuation length at Summit Station in Greenland as a function of depth. 
tance for detecting radio emission from neutrino interactions.

\section{Prototype Instrument}

The GNO prototype instrument includes up to eight dipole antennas. The number of antennas included in the beam-formed array during the 2015 deployment season was tunable. Each antenna consists of two $20 \mathrm{~cm}$ cylinders, each with a diameter of $8 \mathrm{~cm}$. The two sides of the antenna are connected at the feed with 0.635 $\mathrm{cm}$ copper rods that form a cone. The antennas are read out via a $1.27 \mathrm{~cm}$ diameter Heliax cable. A polyvinyl chloride collar separates the two halves of the antenna and provides strain relief at the feed. Feed-to-feed, the antenna spacing was $76.2 \mathrm{~cm}$. An antenna is shown in Fig. 3a.

The antenna frequency range is optimized for the Askaryan emission, the expected RFI environment at Summit, and the diameter of the DISC borehole. The Askaryan signal is broadband up to

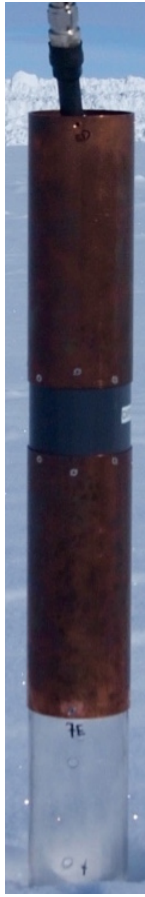

(a)

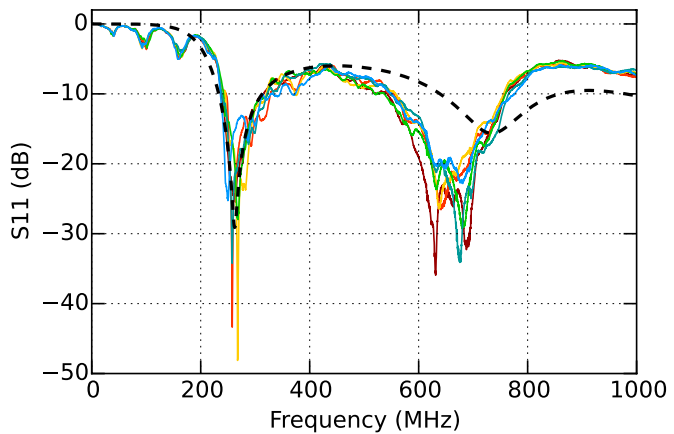

(b)

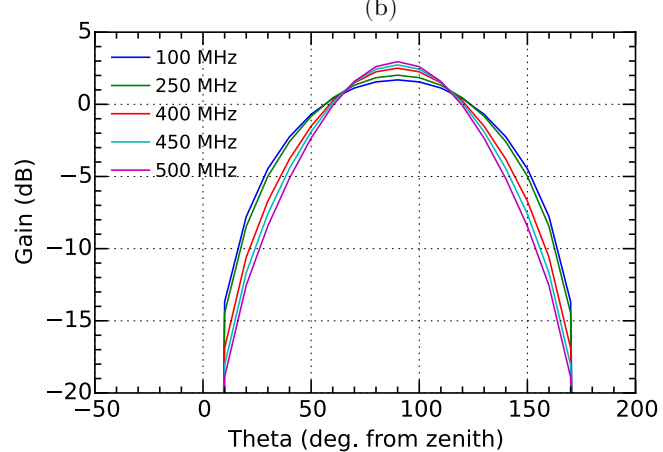

(c)

Figure 3: (a) The dipoles used in the GNO prototype. (b) The return loss for all the antennas used in the array (colored, solid lines) compared with XFDTD (dashed). (c) The predicted beam pattern from XFDTD.

propagation loss at low frequencies, so we preferred antennas in the 100-600 MHz range. The known radio frequency background at Summit Station includes communications frequencies in the 150-160 MHz range and a transmitter at $433 \mathrm{MHz}$. The diameter of the dipoles were chosen to increase the bandwidth, while ensuring that they would still fit within the borehole.

The antenna response, represented by the reflective S-parameter S11 in Fig. 3b, reaches its first resonance at $262 \mathrm{MHz}$ in air, which is reduced to $196 \mathrm{MHz}$ when surrounded by glacial ice $(n=1.78)$. The second dip corresponds to a second mode of the antenna. Simulations of the beam pattern in the horizontal direction with a finite-time difference simulation, XFDTD [17], shown in Fig. 3c, indicate that the second mode results in a slight focusing of the beam pattern with increasing frequency, rather than the formation of an additional null.

Each antenna is amplified by a dual-stage front-end amplifier comprising a $44 \mathrm{~dB}$ amplifier (MITEQ AFS4-00100200-10-15P-4) and a 40 dB amplifier (Mini-Circuits ZKL-1R5) separated by a $3 \mathrm{~dB}$ attenuator. Each front-end amplifier is preceded by a $200 \mathrm{MHz}$ high pass filter to protect the 
first stage amplifier from the $8 \mathrm{MHz}$ transmitter at Summit Station. Coaxial LMR 240 transmits the antenna signal to the surface over $115 \mathrm{~m}$ of cable. Variations in the cable lengths led to a $1 \mathrm{~ns}$ variation in arrival times between antenna channels. Direct current power for the amplifiers is carried through the same cable, coupled and split by bias-tees on the surface and down the borehole.

A schematic of the RF signal chain is shown in Fig. 4. The signal from each antenna is split with $3 \mathrm{~dB}$ couplers into two channels. The first chain samples the signals from the individual chains. The second chain splits the signal eight ways. Each antenna signal travels through fixed delay lengths of LMR 200 that are combined into eight beams at fixed angles from the horizontal: $0^{\circ}, \pm 10^{\circ}, \pm 20^{\circ}, \pm 30^{\circ}$ and $40^{\circ}$. Positive angles correspond to angles pointing from the antenna array to the surface.

Over the 200-300 MHz band, the RF channels vary by $2 \mathrm{~dB}$, as shown in Fig. 5(a). The gains were measured by deconvolving the system response from a fast pulse sent through the signal chain with the

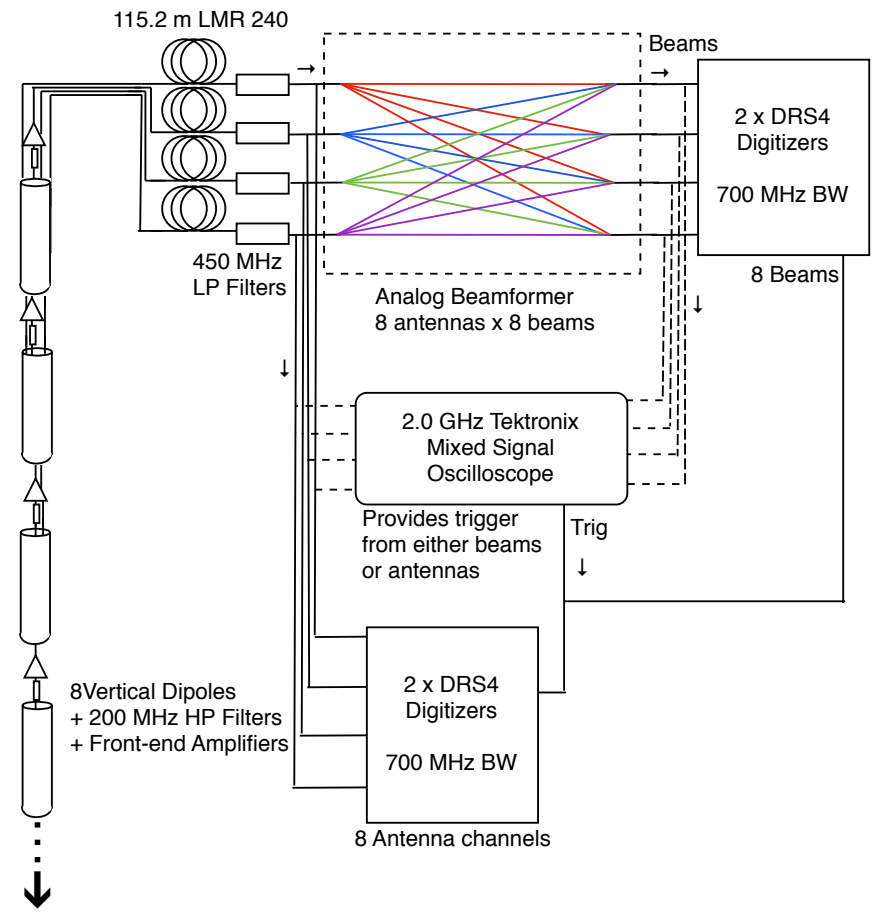

Figure 4: A schematic of the RF chain used in the GNO prototype. Only RF chains and 4 beams are shown for simplicity. injected signal. Fig. 5(b) shows the gain of the beam phased at $0^{\circ}$ from the vertical. Based on the average channel of $68 \mathrm{~dB}$ at $150 \mathrm{MHz}$, the expected beam gain should be $68 \mathrm{~dB}$ after loss in the 8-way power splitter. The

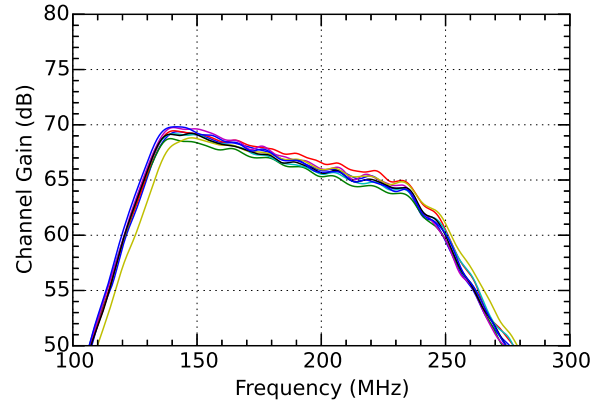

(a) Channel gain

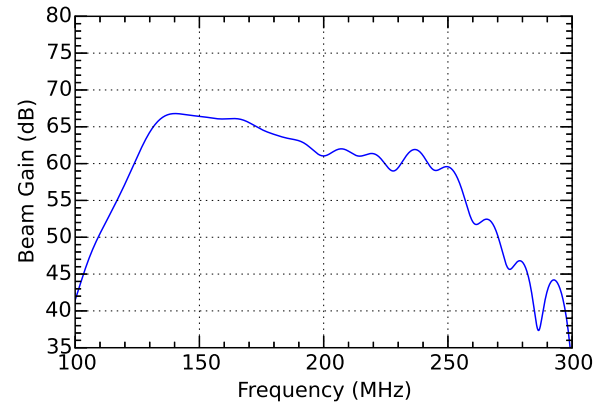

(b) Gain of eight-antennas phased at 0 deg.

Figure 5: Measured gain as a function of frequency for the 8 channels in the phased array (a) before the beam-former and (b) after the beam former for the $0^{\circ}$ beam. 
measured beam gain is $2 \mathrm{~dB}$ lower, consistent with a $1 \mathrm{~ns}$ variation in arrival times through the $\mathrm{RF}$ chains.

Both the antennas and beams are sampled at $2 \mathrm{GSa} / \mathrm{s}$ with record lengths of 1024 points with the DRS4 evaluation boards from the Paul Scherrer Institut [18]. A mixed signal oscilloscope generates a global trigger for the digitizers from a threshold crossing on signals split from either the antennas or beams. The system was powered by batteries for noise measurements, and by a $2 \mathrm{~kW}$ generator for triggered pulse measurements.

The GNO protoype allows for both analog beam-forming through cable delays as well as digital beam-forming of the signal at each antenna. In both cases, the noise is expected to be uncorrelated in each channel, while the signal is correlated. By co-

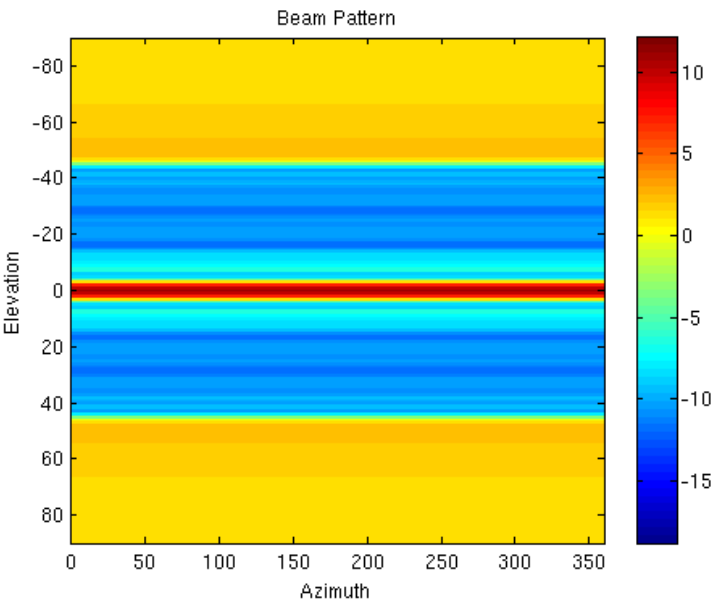

Figure 6: Simulated beam pattern in $\mathrm{dBi}$ of an phased array comprising eight dipoles. herently summing the waveforms from each antenna, the signal amplitude increases linearly, while the noise grows in quadrature, resulting in an increase in the signal-to-noise-ratio (SNR) of $\mathrm{N}$, where $\mathrm{N}$ is the number of antennas included in the phased array. Fig. 6 shows the simulated beam pattern of 8 dipole antennas, each with a gain of $2.15 \mathrm{dBi}$, phased together. The phased beam has a peak gain consistent with a power sum of eight antennas. For such a closely packed array, there exists a second beam angle at which all the antennas phase up at each frequency. This effect is diminished by integrating over the entire band pass.

As an example, we transmitted impulses from a $2 \mathrm{kV}$ pulse generator through a dipole identical to those used in the antenna array. The transmitting antenna was buried in the firn $168 \mathrm{~m}$ from the borehole, and tilted $20^{\circ}$ from the vertical. The signal strength was controlled by attenuating the HV impulse. The pulse width was 1 ns. Fig. 7 shows the measured SNR for two antenna channels for several attenuation settings. By digitally delaying the first channel (RF1) by the time delay expected from both geometry and the timing differences between the RF signal chains on the two channels, the SNR grows by $\sim \sqrt{2}$.

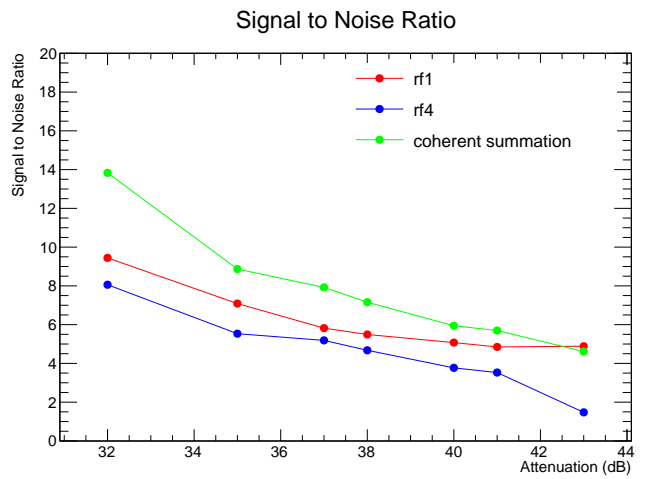

Figure 7: The SNR of impulsive calibration events recorded in two antennas compared with their coherent sum for several pulse strengths.

Fig. 8 shows the time development of the background noise in several channels over several hours one a single night. A monitor antenna placed on the surface (Fig. 8a) is compared with an antenna at deployment depth (Fig. 8b). The surface antenna was amplified by $76 \mathrm{~dB}$ via a $62 \mathrm{~dB}$ 
MITEQ amplifier in line with a $20 \mathrm{~dB}$ attenuator, and a $34 \mathrm{~dB}$ Avtech amplifier. The signal passed through $15 \mathrm{~m}$ of LMR 240 and filters generating a 200-240 MHz bandpass. Analog beams pointed at $0^{\circ}$ and $40^{\circ}$ relative to the horizontal are also shown. At this time, four antennas were included in the array and band-passed between 250 and $450 \mathrm{MHz}$.

During the time shown in Fig. 8, the site was relatively radio quiet, with three noticeable exceptions. A balloon-borne radiosonde launched at Summit Station in support of the ICECAPS atmospheric monitoring program [19] appears in our instrument at $402.8 \mathrm{MHz}$, and the power falls off as the ballon drifts away. Communications lines at $150 \mathrm{MHz}$ also appear as transients in all spectrograms. The transient impulses from a snowmobile are also apparent in the downhole antennas and beams. We note also that during the day, impulses from construction equipment were evident, but we received fewer triggers from them when triggering on the down-going beams.

\section{Conclusions}

We have shown that Summit Station in Greenland is a worldclass site for a neutrino telescope that measures radio emission from neutrino-induced particle showers. This is evident in both the $\sim 1 \mathrm{~km}$ scale attenuation length and the local radio environment. Using a phased array in either Greenland or Antarctica has promise to both improve sensitivity to GZK neutrinos and potentially lower the energy threshold of radio arrays, possibly down to the PeV scale. Our deployment in June 2015 is the first effort towards implementing such an instrument.

\section{Acknowledgements}
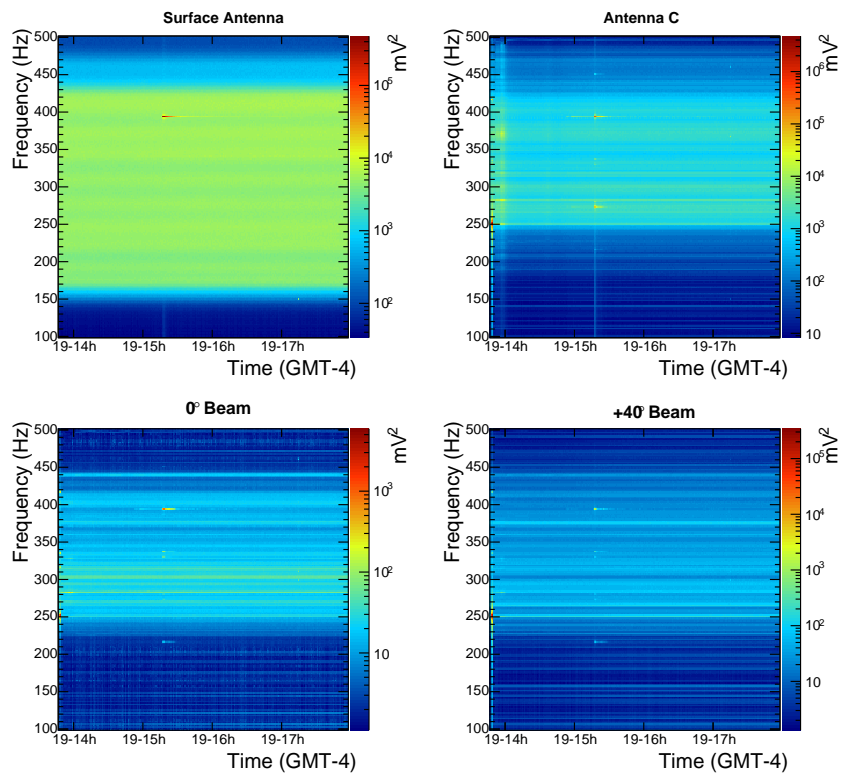

Figure 8: The noise sampled on a surface antenna, downhole antenna, and beams at $0^{\circ}$ and $+40^{\circ}$ from the horizontal.

This work was supported by the Kavli Institute for Cosmological Physics at the University of Chicago, the Department of Energy under Award Number DESC0009937, and others. We would like to thank CH2MHill and the US National Science Foundation (NSF) for their dedicated, knowledgeable, and extremely helpful logistical support team.

\section{References}

[1] V. S. Berezinsky and G. T. Zatsepin, Cosmic rays at ultra high energies (neutrino?), Phys. Lett. B28 (1969) 423. 
[2] G. A. Askaryan, Excess Negative Charge of the Electron-Photon Shower and Coherent Radiation Originating from It, JETP 14 (1962) 441.

[3] G. A. Askaryan, Coherent Radio Emission from Cosmic Showers in Air and in Dense Media, JETP 21 (1965) 658.

[4] D. P. Saltzberg, et al., Observation of the Askaryan Effect: Coherent Microwave Cherenkov Emission from Charge Asymmetry in High-Energy Particle Cascades, PRL 86 (2001), 2802.

[5] P. W. Gorham, et al. Accelerator measurements of the Askaryan effect in rock salt: A roadmap toward teraton underground neutrino detectors, PRD 72 (2005), 023002.

[6] P. W. Gorham. Observations of the Askaryan Effect in Ice, PRL 99 (2007) 171101.

[7] P. W. Gorham, et al., The Antarctic Impulsive Transient Antenna ultra-high energy neutrino detector: Design, performance, and sensitivity for the 2006-2007 balloon flight Astropart. Phys. 32 (2009), 10.

[8] ARA Collaboration, P. Allison, et al. First Constraints on the Ultra-High Energy Neutrino Flux from a Prototype Station of the Askaryan Radio Array, Astropart. Phys. 70 (2015) 62.

[9] S. Barwick, et al. Design and Performance of the ARIANNA Hexagonal Radio Array Systems (2014) [astro-ph. IM:1410.7369].

[10] A. G. Vieregg, K. Bechtol, A. Romero-Wolf. A New Technique for Detection of PeV Neutrinos Using a Phased Radio Array, submitted Astropart. Phys. (2015) [astroph. IM/1504.08006v1]

[11] K. Bechtol, A. G. Vieregg, A. Romero-Wolf, and S. Wissel. A New Trigger for Detection of PeV to EeV Neutrinos Using a Phased Radio Array, These proceedings (2015).

[12] The IceCube Collaboration, M. G. Aartsen, et al., Observation of High-Energy Astrophysical Neutrinos in Three Years of IceCube Data, Physical Review Letters 113 (2014) 101101.

[13] J. Avva, J. M. Kovac, C. Miki, D. Saltzberg, A. Vieregg, An in situ measurement of the radio-frequency attenuation in ice at Summit Station, Greenland, submitted Journal of Glaciology (2015) [astroph. IM/1409.5413v2].

[14] D. Z. Besson, et al., In situ radioglaciological measurements near Taylor Dome, Antarctica and implications for ultra-high energy (UHE) neutrino astronomy, Astropart. Physics 29 (2008) 130.

[15] S. Barwick, et al., South Polar in situ radio-frequency ice attenuation, Journal of Glaciology 51 (2005) 231.

[16] T. Barrella, S. Barwick, and D. Saltzberg, Ross Ice Shelf (Antarctica) in situ radio-frequency attenuation, Journal of Glaciology 57 (2011) 61.

[17] K. Kunz and R. Luebbers. The Finite Difference Time Domain Method for Electromagnetics, CRC Press (1993).

[18] S. Ritt, Development of high speed waveform sampling ASICs, In Proc. NSNI (2010).

[19] M. Shupe, et al., High and Dry: New Observations of Tropospheric and Cloud Properties above the Greenland Ice Sheet, Bull. Amer. Metero. Soc. 94 (2013), 169. 